\title{
ON THE OPERATIONAL CALCULUS FOR GROUPS OF OPERATORS ${ }^{1}$
}

\section{SHMUEL KANTOROVITZ}

ABstract. We study some compactness properties of the natural operational calculus for groups of operators.

1. Let $\{T(t) \mid t$ real $\}$ be a strongly continuous group of operators on a Banach space $X$. Let $M_{T}$ be the class of all complex Borel measures $m$ on the real line $R$ such that

$$
\|m\|_{T}=\int\|T(t)\| d|m|(t)<\infty .
$$

$M_{T}$ is a Banach algebra for the usual operations with measures and for the norm $\|\cdot\|_{T}$. The image $A_{T}$ of $M_{T}$ under the Fourier transform $m \rightarrow \hat{m}$ is a Banach algebra of bounded continuous complex functions on $R$, with the usual pointwise operations and with the norm $\|\hat{m}\|_{T}$ $=\|m\|_{T}$.

If $m_{t}$ denotes the delta measure at $t$ and $f_{t}(s)=\hat{m}_{t}(s)=\exp (i t s)$ $(t, s \in R)$, then $m_{t} \in M_{T}\left(f_{t} \in A_{T}\right)$ and

$$
\left\|f_{t}\right\|_{T}=\left\|m_{t}\right\|_{T}=\|T(t)\|, \quad t \in R .
$$

Let $F$ be a Banach algebra of complex functions on $R$ which contains the functions $f_{t}(t \in R)$. An $F$-operational calculus for $T(\cdot)$ is a continuous representation of $F$ on $X$ which sends $f_{t}$ to $T(t)$ for all $t \in R$.

For $f \in A_{T}$, define the operator $\tau(f)$ on $X$ by

$$
\tau(f) x=\int T(t) x d m(t) \quad\left(x \in X, f=\hat{m} \in A_{T}\right) .
$$

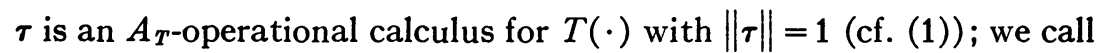
it the natural operational calculus for $T(\cdot)$.

The operational calculus may be localized as follows. For $0 \neq x \in X$,

Received by the editors March 3, 1970.

AMS 1969 subject classifications. Primary 4730; Secondary 4750.

Key words and phrases. Group of operators, operational calculus, compact operator, compactly imbedded, regular vectors, spectral operator, quasi-nilpotent operator.

1 Research supported by NSF Grant GP 8289. 
let $A_{T x}$ denote the Banach space of all Fourier transforms of complex Borel measures $m$ on $R$ for which

$$
\|\hat{m}\|_{T x}=\int\|T(t) x\| d|m|(t)<\infty,
$$

with the norm $\|\cdot\|_{T x}$. The map $\tau(\cdot) x: A_{T_{x}} \rightarrow X$ defined by (2) is a continuous linear mapping of norm 1 , such that $\tau\left(f_{t}\right) x=T(t) x$ and $\tau(f g) x=\tau(f) \tau(g) x\left(f \in A_{T}, g \in A_{T x}\right)$.

Definition 1. $x \in X$ is (strongly) regular for $T(\cdot)$ if the ratio $\|T(\cdot) x\| /\|T(\cdot)\|$ has a positive (infimum) lim sup as $|t| \rightarrow \infty$.

THEOREM 1. Let $S(\cdot)$ and $T(\cdot)$ be strongly continuous groups of operators, and let $x \in X$. Then

(i) $A_{S} \subset A_{T}$ iff $\|T(\cdot)\| /\|S(\cdot)\|$ is bounded.

(ii) $A_{T x}=A_{T}$ iff $x$ is strongly regular for $T(\cdot)$.

Proof. Let $C_{0}^{\prime}$ be the dual of the space $C_{0}$ of all complex continuous functions with compact support on $R$ (with the usual topology). If $h \in C_{0}, Q=\operatorname{supp} h$, and $K=\sup _{Q}\|T(\cdot)\|^{-1}$ (finite!), then

$$
\left|\int h d m\right| \leqq K \sup |h| \cdot\|m\|_{T},
$$

so that $M_{T}$ is topologically contained in $C_{0}^{\prime}$. Now if $A_{S} \subset A_{T}$, then $M_{S} \subset M_{T}$, and the latter (hence the former) inclusion is topological, since both spaces are Banach spaces topologically contained in $C_{0}^{\prime}$ (cf. the Closed Graph Theorem). Since $f_{t} \in A_{s}$, it follows that

$$
\|T(t)\|=\left\|f_{t}\right\|_{T} \leqq B\left\|f_{t}\right\|_{S}=B\|S(t)\|, \quad t \in R .
$$

This proves the nontrivial part of (i), and (ii) is proved by the same argument.

2. The group $T(\cdot)$ is bounded away from zero if inf $\|T(\cdot)\|>0$, and temperate if $\|T(t)\|=O\left(|t|^{k}\right)$ as $|t| \rightarrow \infty$, for some nonnegative integer $k$. Let

$$
w^{ \pm}=\lim _{t \rightarrow \pm \infty} t^{-1} \log \|T(t)\|
$$

(cf. [2, p. 244]). The spectral norm of $T(t)$ is $\exp \left[t w^{+}\right]$for $t \geqq 0$ and $\exp \left[t w^{-}\right]$for $t<0$; this implies the equivalence of the following statements:

1. $T(\cdot)$ is bounded away from zero;

2. $\|T(\cdot)\| \geqq 1$;

3. $w^{-} \leqq 0 \leqq w^{+}$. 
In particular, temperate groups are bounded away from 0.

For $Q \subset R$ compact, denote by $A_{T}(Q)$ the closed subspace of $A_{T}$ consisting of all $f \in A_{T}$ with support in $Q$. If $f=\hat{m} \in A_{T}(Q)$, then $d m(t)=\hat{f}(-t) d t / 2 \pi$, and therefore

$$
\|f\|_{T}=\int\|T(t)\||\hat{f}(-t)| d t / 2 \pi, \quad f \in A_{T}(Q) .
$$

THEOREM 2. Let $S(\cdot)$ and $T(\cdot)$ be strongly continuous groups of operators. If $S(\cdot)$ is bounded away from 0 and $\|T(t)\| /\|S(t)\| \rightarrow 0$ as $|t| \rightarrow \infty$, then, for each compact $Q \subset R$ and $x \in X, A_{S}(Q)$ is compactly imbedded in $A_{T}$ and the restrictions $\tau \mid A_{S}(Q)$ and $\tau(\cdot) x \mid A_{S}(Q)$ are compact operators. Conversely, if $S(\cdot)$ and $T(\cdot)$ are temperate and $A_{S}(Q)$ is compactly imbedded in $A_{T}$ for some compact $Q$ with nonvoid interior, then $\|T(t)\| /\|S(t)\| \rightarrow 0$ as $|t| \rightarrow \infty$.

Proof. 1. Let $S(\cdot)$ be bounded away from 0 (hence $\|S(\cdot)\| \geqq 1$ ), and let $\|T(t)\| /\|S(t)\| \rightarrow 0$ as $|t| \rightarrow \infty$. Given $\epsilon>0$, choose $N>0$ such that $\|T(t)\| /\|S(t)\|<\epsilon$ for $|t|>N$. Let $Q \subset R$ be compact, and let $\left\{h_{n}^{\prime}\right\}$ be in the closed unit ball of $A_{S}(Q)$. By (3), $\left\{\hat{h}_{n}^{\prime}\right\}$ is in the closed $2 \pi$-ball of $L^{1}(R)$. Therefore, a subsequence $\left\{\hat{h}_{n}\right\}$ converges weak* in $\left(L^{\infty}\right)^{*}$. In particular, for each $s \in R$

$$
\frac{1}{2 \pi} \int e^{-i t s} \hat{h}_{n}(t) d t=h_{n}(s)
$$

converge. Since

$$
\left|h_{n}(s)\right| \leqq \frac{1}{2 \pi}\left\|\hat{h}_{n}\right\|_{L^{1}} \leqq 1 \text { and } \hat{h}_{n}(t)=\int_{Q} e^{i t s} h_{n}(s) d s,
$$

it follows by Dominated Convergence that $\hat{h}_{n}$ converge pointwise on $R$ and $\left|\hat{h}_{n}\right| \leqq|Q|$ (the Lebesgue measure of $Q$ ). Now, by (3),

$$
\left\|h_{n}-h_{m}\right\|_{T} \leqq \int_{-N}^{N}\|T(t)\|\left|\hat{h}_{n}(-t)-\hat{h}_{m}(-t)\right| d t / 2 \pi+\epsilon\left\|h_{n}-h_{m}\right\|_{s} .
$$

The first term on the right tends to 0 as $n, m \rightarrow \infty$ (Dominated Convergence), and the second term is $\leqq 2 \epsilon$. Hence $\left\{h_{n}\right\}$ is Cauchy in $A_{T}$, and the first part of the theorem follows.

2. Let $S(\cdot)$ and $T(\cdot)$ be temperate, and suppose $A_{S}(Q)$ is compactly imbedded in $A_{T}$ for some compact $Q$ with nonvoid interior. There exists a nonzero $C^{\infty}$ function $g$ with support in $Q$. Let $g_{t}=$ $g f_{t} /\|S(t)\|(t \in R)$. Then $g_{t} \in A_{S}(Q)$, and by (3), 


$$
\begin{aligned}
\left\|g_{t}\right\|_{S} & =\int\|S(s)\||\hat{g}(t-s)|\|S(t)\|^{-1} d s / 2 \pi \\
& =\int\|S(t+u)\|\|S(t)\|^{-1}|\hat{g}(-u)| d u / 2 \pi \\
& \leqq \int\|S(u)\||\hat{g}(-u)| d u / 2 \pi=\|g\|_{s} \quad(t \in R) .
\end{aligned}
$$

Therefore, the set $\left\{g_{t} \mid t \in R\right\}$ has limit points in $A_{T}$, which are also limit points in the topology induced on $A_{T}$ by the weak* topology on $L^{\infty}(R)$ (because $\|T(\cdot)\| \geqq 1$ ). However, $g_{t} \rightarrow 0$ (as $\left.|t| \rightarrow \infty\right)$ in the latter topology, since for each $h \in L^{1}(R)$,

$$
\left|\int h(s) g_{t}(s) d s\right|=|(h g) \hat{(}(t)| /|| S(t)|| \leqq(h g) \hat{(}(t) \mid \rightarrow 0
$$

by the Riemann-Lebesgue lemma. Therefore $g_{t} \rightarrow 0$ in $A_{T}$ as $|t| \rightarrow \infty$. Now

$$
\begin{aligned}
\left\|g_{t}\right\|_{r} & =\int\|T(s)\||\hat{g}(t-s)| d s /(2 \pi\|S(t)\|) \\
& \geqq(\|T(t)\| /\|S(t)\|) \int\|T(t-s)\|^{-1}|\hat{g}(t-s)| d s / 2 \pi \\
& =c\|T(t)\| /\|S(t)\|,
\end{aligned}
$$

where $c=\int\|T(u)\|^{-1}|\hat{g}(u)| d u / 2 \pi$ is a positive constant. Since $\left\|g_{t}\right\|_{T \rightarrow 0}$, the result follows.

The same arguments (with the estimate $\|T(s) x\| \geqq\|T(t) x\|$ - $\|T(t-s)\|^{-1}$ replacing the inequality $\|T(s)\| \geqq\|T(t)\|\|T(t-s)\|^{-1}$ in the last part of the proof) prove the following.

THEOREM 3. Let $T(\cdot)$ be a strongly continuous group of operators, and let $x \in X$. If $T(\cdot)$ is bounded away from zero and $x$ is nonregular, then $A_{T}(Q)$ is compactly imbedded in $A_{T x}$ for each compact $Q \subset R$. Conversely, if $T(\cdot)$ is temperate and $A_{T}(Q)$ is compactly imbedded in $A_{T x}$ for some compact set $Q$ with nonvoid interior, then $x$ is nonregular.

CoROllary. Let $T(\cdot)$ be a temperate strongly continuous group of operators. Then $x$ is a regular vector for $T(\cdot)$ iff the injection $A_{T}(Q)$ $\rightarrow A_{T_{x}}$ is not compact for any compact set $Q$ with nonvoid interior.

3. Remarks. The existence of regular vectors seems to be a difficult question in general. Observe that if $S(\cdot)$ and $N(\cdot)$ are commuting 
groups of operators, and $S(\cdot)$ is uniformly bounded, then the groups $T(\cdot)=S(\cdot) N(\cdot)$ and $N(\cdot)$ have the same (strongly) regular vectors. This follows from the estimate (with $K=\sup _{R}\|S(\cdot)\|$ ):

$$
\|T(t) x\| /\|T(t)\| \geqq K^{-1}\|S(t) N(t) x\| /\|N(t)\| \geqq K^{-2}\|N(t) x\| /\|N(t)\| .
$$

In particular, every nonzero vector is strongly regular for a uniformly bounded group.

Proposition. If $T$ is a real spectral operator of finite type, then the group $T(t)=e^{i t T}$ possesses regular vectors.

Proof. Let $T=S+N$ be the canonical decomposition of $T$ (cf. [1]). Then $T(\cdot)=S(\cdot) N(\cdot)$, where $S(t)=e^{i t s}$ and $N(t)=e^{i t N}$ are commuting groups and $S(\cdot)$ is uniformly bounded. By the preceding remark, it suffices to show that $N(\cdot)$ possesses regular vectors. Let $k$ be the nonnegative integer defined by the requirements $N^{k} \neq 0$ and $N^{k+1}=0$. Then

$$
(i t)^{-k} N(t)=\sum_{j=0}^{k-1}(i t)^{j-k} N^{j} / j !+N^{k} / k ! \rightarrow N^{k} / k !
$$

as $|t| \rightarrow \infty$, in the uniform operator topology. Therefore,

$$
\begin{aligned}
\lim _{|t| \rightarrow \infty}\|N(t) x\| /\|N(t)\| & =\lim _{|t| \rightarrow \infty}\left\|(i t)^{-k} N(t) x\right\| /\left\|(i t)^{-k} N(t)\right\| \\
& =\left\|N^{k} x\right\| /\left\|N^{k}\right\|,
\end{aligned}
$$

so that each vector not in the null space of $N^{k}$ is regular for $N(\cdot)$.

CONJECTURE. Every temperate group of operators possesses regular vectors.

The following example of R. Beals shows that the "finite type" hypothesis cannot be omitted in the above proposition.

For $n=1,2, \cdots$, let $N_{n}$ be the $n \times n$ matrix with $1 / n$ in the first superdiagonal and 0 elsewhere, and consider $N=\sum \bigoplus N_{n}$ as an operator in $l_{1}$. Since $N_{n}^{n}=0$ and $\left\|N_{n}^{k}\right\|=n^{-k}$ for $k<n$, we have $N^{k}$ $=\sum_{n>k} \oplus N_{n}^{k}$ and $\left\|N^{k}\right\|=\sup _{n>k} n^{-k}=(k+1)^{-k}$. Hence $N$ is quasinilpotent. Let $N(t)=e^{t N}=\sum \bigoplus \exp \left(t N_{n}\right)$. One calculates that $\left\|\exp \left(t N_{n}\right)\right\|=\sum_{k<n}(t / n)^{k} / k$ !. Since

$$
\|N(t)\|=\sup _{n}\left\|\exp \left(t N_{n}\right)\right\|
$$

it follows that for each fixed $n$,

$$
\left\|\exp \left(t N_{n}\right)\right\| /\|N(t)\| \leqq\left\|\exp \left(t N_{n}\right)\right\| /\left\|\exp \left(t N_{n+1}\right)\right\| \rightarrow 0
$$

as $t \rightarrow \infty$. 
Let $P_{n}:\left(x_{1}, x_{2}, \cdots\right) \rightarrow\left(x_{1}, \cdots, x_{m(n)}, 0,0, \cdots\right)$, where $m(n)$ $=1+\cdots+n$. For any $x \in l_{1}$ and $n=1,2, \cdots$,

$$
\begin{aligned}
\|N(t) x\| /\|N(t)\| & \leqq\left\|N(t) P_{n} x\right\| /\|N(t)\|+\left\|N(t)\left(I-P_{n}\right) x\right\| /\|N(t)\| \\
& \leqq\left\|\exp \left(t N_{n}\right)\right\|\|x\| /\|N(t)\|+\left\|\left(I-P_{n}\right) x\right\|,
\end{aligned}
$$

and therefore,

$$
\limsup _{t \rightarrow \infty}\|N(t) x\| /\|N(t)\| \leqq\left\|\left(I-P_{n}\right) x\right\|,
$$

by (1). Letting $n \rightarrow \infty$, we obtain that the lim sup is 0 . Similarly, the lim sup as $t \rightarrow-\infty$ is 0 . Since $x$ was arbitrary, this shows that $N(\cdot)$ has no regular vectors.

\section{REFERENCES}

1. N. Dunford, $A$ survey of the theory of spectral operators, Bull. Amer. Math. Soc. 64 (1958), 217-274. MR 21 \#3616.

2. E. Hille and R. S. Phillips, Functional analysis and semi-groups, rev. ed., Amer. Math. Soc. Colloq. Publ., vol. 31, Amer. Math. Soc., Providence, R. I., 1957. MR 19, 664.

University of Illinois at Chicago Circle, Chicago, Illinois 60680 